\title{
HIGHLIGHTS
}

ENDOSCOPY

\section{Snoring while under conscious sedation during colonoscopy is linked to obstructive sleep apnea}

Snoring while under conscious sedation during colonoscopy is a strong predictor of obstructive sleep apnea (OSA), according to a recent study by Ala Sharara et al.

"Although snoring is commonly noted in patients during colonoscopy, this observation had not been adequately addressed or scientifically investigated," explains Sharara. "The question arose as to whether such patients have undiagnosed OSA, a condition associated with significant morbidity, mortality and affiliated costs."

131 consecutive patients undergoing colonoscopy with conscious sedation at the authors' center completed a preendoscopy sleep questionnaire and a physical examination that was designed to detect OSA. Patients who snored for $\geq 10 \mathrm{~s}$ during colonoscopy underwent ambulatory polysomnography. This procedure was also performed in matched controls who

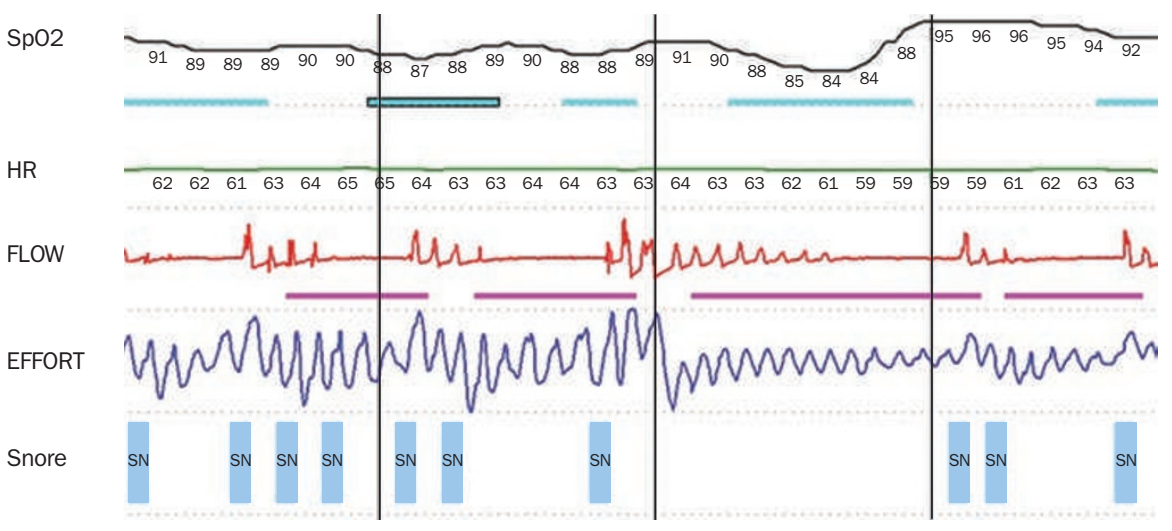

A polysomnogram, courtesy of A. Sharara.

did not snore. All 20 patients who snored during colonoscopy and were evaluated by polysomnography had evidence of OSA compared with 4 of 18 controls. Moderate or severe OSA was revealed in 14 of the 20 snorers (70\%), versus 1 of the 18 controls (5.6\%). Compared with snoring under conscious sedation, pre-endoscopy physical examination and sleep questionnaire failed to accurately predict OSA.

"Conscious sedation may unmask OSA," says Sharara. "The next step will be to see whether portable OSA monitoring performed during endoscopy is sensitive and specific enough to detect OSA. With the obesity epidemic, the number of patients with undiagnosed OSA is expected to grow so careful evaluation of individuals undergoing colonoscopy could help uncover this morbid condition."

\section{Rachel Jones}

Original article Sharara, A. I. et al. Persistent snoring under conscious sedation during colonoscopy is a predictor of obsructive sleep apnea. Gastrointest. Endosc. 71, 1224-1230 (2010). 